\title{
Impact of Pregnancy on Hidradenitis Suppurativa Disease Course: A Systematic Review and Meta-Analysis
}

\author{
Justine R. Seivright ${ }^{a} \quad$ Natalie M. Villab ${ }^{b}$ Tristan Groganc ${ }^{c}$ Ram K. Parvataneni ${ }^{d}$ \\ Alyssa M. Thompson ${ }^{\text {e }}$ Vivian Y. Shi ${ }^{f}$ Jennifer L. Hsiao ${ }^{b}$

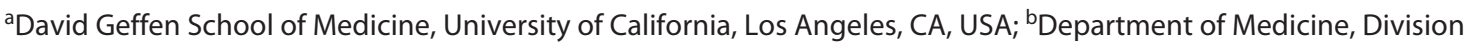 \\ of Dermatology, University of California Los Angeles, Los Angeles, CA, USA; 'Department of Medicine Statistics Core,

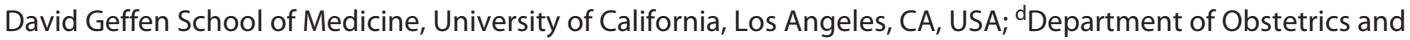 \\ Gynecology, University of California, Los Angeles, CA, USA; eUniversity of Arizona College of Medicine, Tucson, AZ, \\ USA; fDepartment of Dermatology, University of Arkansas for Medical Sciences, Little Rock, AR, USA
}

\section{Keywords}

Hidradenitis suppurativa · Pregnancy · Disease course ·

Postpartum flare $\cdot$ Acne inversa

\begin{abstract}
Background: Hormones are thought to play a role in hidradenitis suppurativa (HS). However, data on the HS disease course during pregnancy and the postpartum period has not been well established. The objective of this study is to analyze the available literature to determine HS disease activity during pregnancy and the postpartum period. Methods: The PubMed and Embase databases were systematically searched for relevant articles from database inception until November 22, 2020. The inclusion criteria were a study population with the diagnosis of HS and discussion of pregnancy impact on the HS disease course or postpartum flare. Study characteristics, patient demographics, HS severity, and HS disease course during pregnancy and the postpartum period were extracted by 2 independent reviewers. The quality of included studies was assessed using the Newcastle-Ottawa Scale for observational studies. Heterogeneity was assessed using Cochran's $Q$ statistic and $P$ index. The randomeffects meta-analytical model was used. The primary study outcome was the pooled odds ratio of improvement or of
\end{abstract} \\ Karger}

worsening of HS disease activity during pregnancy. Results: The systematic search identified 8 studies for analysis. There was a total of 672 cases for which data on the patient-reported HS disease course during pregnancy were available, and 164 cases for which data on patient-reported postpartum flare were available. In the meta-analyses, the rate of HS disease improvement was $24 \%(95 \% \mathrm{Cl} 0.13-0.40)$ and the rate of HS disease worsening was $20 \%(95 \% \mathrm{Cl} 0.11-0.34)$. Sixty percent $(99 / 164)$ of patients experienced a postpartum flare. Conclusion: While about a quarter of women will experience an improvement in HS during pregnancy, the majority will have a stable or worsened disease course, and over half of patients will experience a postpartum flare. Close monitoring of HS patients is needed during pregnancy and postpartum periods, as patients may need continued, or even escalated, disease management.

(c) 2021 S. Karger AG, Basel

\section{Introduction}

Hidradenitis suppurativa (HS) is a chronic and debilitating dermatosis characterized by painful draining nodules and sinus tracts typically in the intertriginous areas. HS disproportionately affects women, particularly those 
Fig. 1. PRISMA flow diagram. From Moher et al. [24] (www.prisma-statement.org).

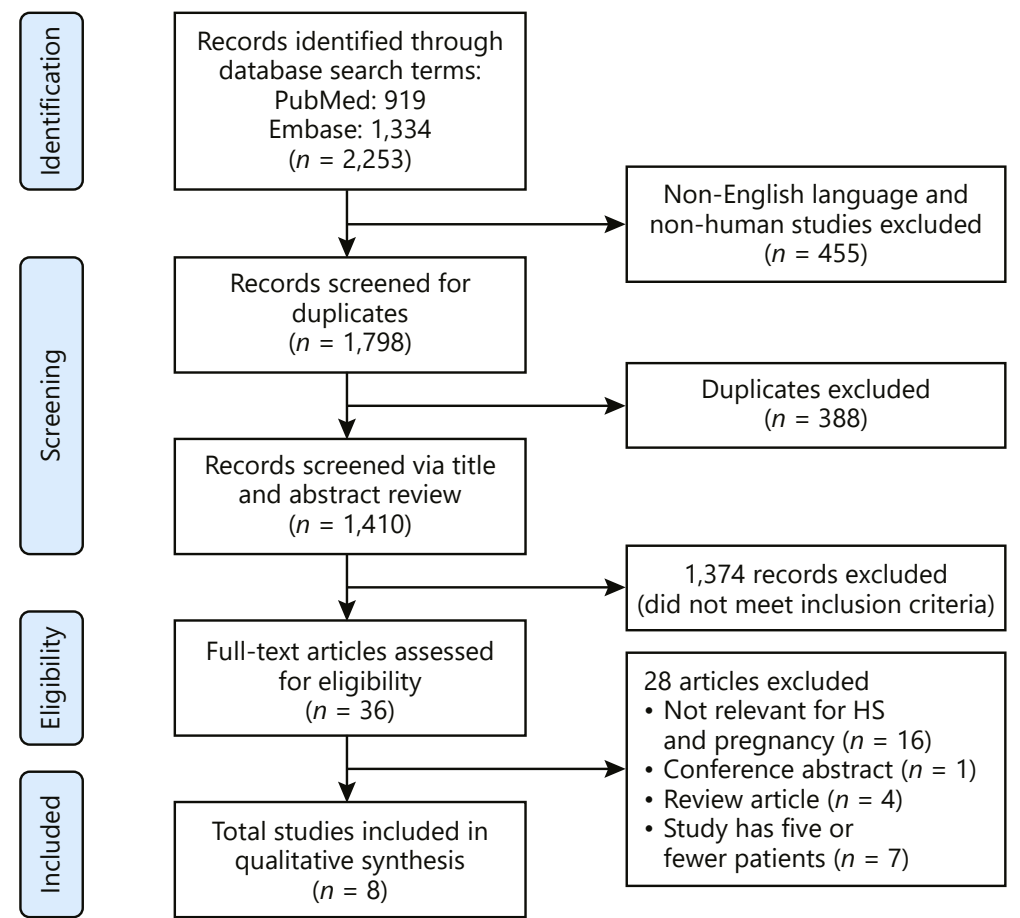

of childbearing age [1]. Hormones are thought to play a role in HS pathogenesis, as supported by the presence of perimenstrual HS disease flares [2]. However, data on the impact of pregnancy on the HS disease course has been mixed. Although some studies have found that HS activity may improve during pregnancy [3-5], others have reported disease worsening $[5,6]$. Comprehensive information about the impact of pregnancy on HS disease activity is essential to aid informed decision making during pre-pregnancy counseling, and to plan for adequate monitoring and follow-up during pregnancy and after delivery. Herein we systematically review the literature and perform a meta-analysis to examine the impact of pregnancy on the HS disease course. This study aims to determine: (1) whether HS disease activity improves or worsens during pregnancy, and (2) the frequency of postpartum HS flare.

\section{Methods}

\section{Search Strategy}

A systematic review and meta-analysis was performed following the Preferred Reporting Items for Systematic Reviews and Meta-Analyses (PRISMA) guidelines to investigate whether HS im- proves or worsens during pregnancy. The PRISMA flow diagram with the search schema is shown in Figure 1. The PubMed and Embase databases were accessed using the following search terms: "hidradenitis suppurativa" OR "hidradenitis" OR "acne inversa" OR "Velpeau disease" OR "Verneuil disease" AND "pregnant" OR "pregnancy" OR "gestation" OR "conception" OR "childbirth" OR "delivery” OR "woman” OR "women” OR “worsen” OR "deterioration" OR "exacerbation" OR "flare" OR "trigger" OR "amelioration" OR “improvement” OR "remission" OR "postpartum." The search included all articles from database inception to the date our search was performed on November 22, 2020. A total of 2,253 articles were identified. Articles were filtered to remove non-English language and non-human studies, which resulted in 1,798 studies. After duplicates were removed, 1,410 articles remained.

The title and abstract of the 1,410 identified articles were independently screened for relevance by 2 authors (J.R.S. and N.M.V.), with any discrepancies discussed to a consensus. The inclusion criteria were a study population with the diagnosis of HS and discussion of pregnancy impact on the disease course or postpartum flare. Thirty-six articles were included for full-text review. Conference abstracts, reviews or commentaries with no original data, studies containing data for 5 or fewer patients, and non-relevant articles were excluded. After full-text review, another 28 articles were excluded. Articles selected for full-text review were also screened for references that fit the inclusion criteria, but no additional relevant articles were identified. Eight articles were included in the final data analysis. 


\section{Data Extraction}

Data extraction was independently performed by 2 reviewers (J.R.S. and N.M.V.). For each article, the 2 reviewers recorded the study design, country of study, patient demographics, HS severity, patient-reported HS disease course during pregnancy, and patient-reported presence or absence of postpartum flare. The criteria used by each study for assessing change in HS disease activity were recorded. Quality assessment of the studies was independently performed by both reviewers using the Newcastle-Ottawa Scale (NOS) for observational studies and the modified version for cross-sectional studies [7, 8]. Any discrepancies were discussed to a consensus with a third reviewer (J.L.H.). No contact with any of the included studies' authors was required.

\section{Meta-Analyses}

Two meta-analyses were conducted to assess patient-reported: (1) HS disease improvement, and (2) HS disease worsening during pregnancy. Forest plots were constructed using the proportion of women with improved or with worsened symptoms and standard errors/confidence intervals were computed using inverse variance weighting. Heterogeneity was assessed using Cochran's Q statistic and $I^{2}$ index (the percentage of variation across studies, i.e., due to heterogeneity rather than chance) [9]. Because significant heterogeneity was observed for both analyses, the random-effects meta-analytical model was utilized as opposed to the fixed-effects pooled estimate. Statistical analyses were performed using R v.3.6.1 (www.rproject.org) utilizing the "meta" package. $p$ values $<0.05$ were considered statistically significant. A sensitivity analysis that included relevant studies with 5 or fewer patients was also performed to see if the pooled estimates changed much when they were included.

\section{Results}

This systematic review and meta-analysis included 8 articles published between 1986 and 2020 that reported HS disease courses during pregnancy. A total of 672 cases were identified for which data on HS disease course during pregnancy were available. Demographic data including age, race, and disease severity are summarized in Table 1 . The mean age at the time of study ranged from 31 to 53.7 years $[3-5,10,11]$. Race was reported in only 2 studies $[5,6]$. Two studies included HS severity data at the time of study, and both included predominantly Hurley II and Hurley III patients (67.4-90.6\%) [3, 5]. Lyons et al. [6] reported demographic and HS severity information at the time of pregnancy; the mean age was 25.9 years, and out of 191 pregnancies, about half (53.9\%) were in patients with baseline Hurley I disease.

Study locations included the UK $(n=3)$, the USA ( $n=$ $2)$, Denmark $(n=2)$, and the Netherlands $(n=2) ; 1$ study was conducted in both Denmark and the Netherlands [4], and 1 study distributed electronic surveys to international support groups [5]. There were 6 cross-sectional studies, 1 case-control study, and 1 retrospective cohort study.
Of a maximum of 10 points for cross-sectional studies using the modified NOS cross-sectional scale, quality scores ranged from 3 to 7 . Of a maximum of 9 points for cohort and case-control studies, both studies scored 5 .

All studies used patient report as the criteria for assessing change in HS disease activity during pregnancy and postpartum, where reported. In 7 studies, individuals were asked in a survey or interview to describe the effect of pregnancy on their HS disease course; in 1 study, patient report of disease activity during pregnancy was extracted via a retrospective chart review and number of cases was based on individual pregnancies [6]. Fernandez et al. [5] reported data on differences in delivery methods. Lyons et al. [6] included information regarding HS treatment during pregnancy. None of the studies included data on the specific timing of HS disease improvement or worsening during pregnancy, such as trimester-specific information.

\section{HS Disease Course during Pregnancy}

Of the 672 cases summarized in Table 1, disease activity improved in 185 (28\% overall) and varied from $0 \%$ $(0 / 17)$ [12] to $83 \%(5 / 6)$ [11] across the studies. Based on the meta-analysis of pooled data from all 8 studies, the patient-reported rate of HS improvement was 0.24 (95\% CI 0.13-0.40). Significant heterogeneity exists between the studies $\left(I^{2}=92 \%\right.$; Fig. $\left.2 \mathrm{a}\right)$. Disease activity worsened in 205 (31\% overall) of the 672 cases; this varied from $0 \%$ (0/6) [11] to $62 \%(70 / 113)$ [6] across the studies. Based on our meta-analysis, the patient-reported rate of HS disease worsening was 0.20 (95\% CI $0.11-0.34)$, with a large amount of heterogeneity observed $\left(I^{2}=91 \%\right.$; Fig. $\left.2 b\right)$. As a sensitivity analysis, we also included the 7 identified studies with 5 or fewer patients. The pooled estimate changed to 0.29 (95\% CI 0.15-0.48) for disease improvement and to 0.23 (95\% CI 0.13-0.38) for disease worsening, showing similar results to our meta-analyses above.

\section{Postpartum HS Disease Course}

Five studies in our systematic review investigated patient-reported HS disease activity during the postpartum period (Fig. 3). Four of the 5 studies quantified general postpartum disease activity; a worsening in disease activity during the postpartum period was noted in 99/164 $(60 \%)$ cases $[6,10-12]$. The fifth study reported postpartum HS flare in the specific context of vaginal delivery; 38/162 (23.5\%) women with anogenital HS who delivered vaginally believed that vaginal delivery caused an HS flare [5]. The timeframe considered to be the postpartum period was not defined by any of the studies except for Lyons et al. [6], defined as the first 6 months after delivery. 


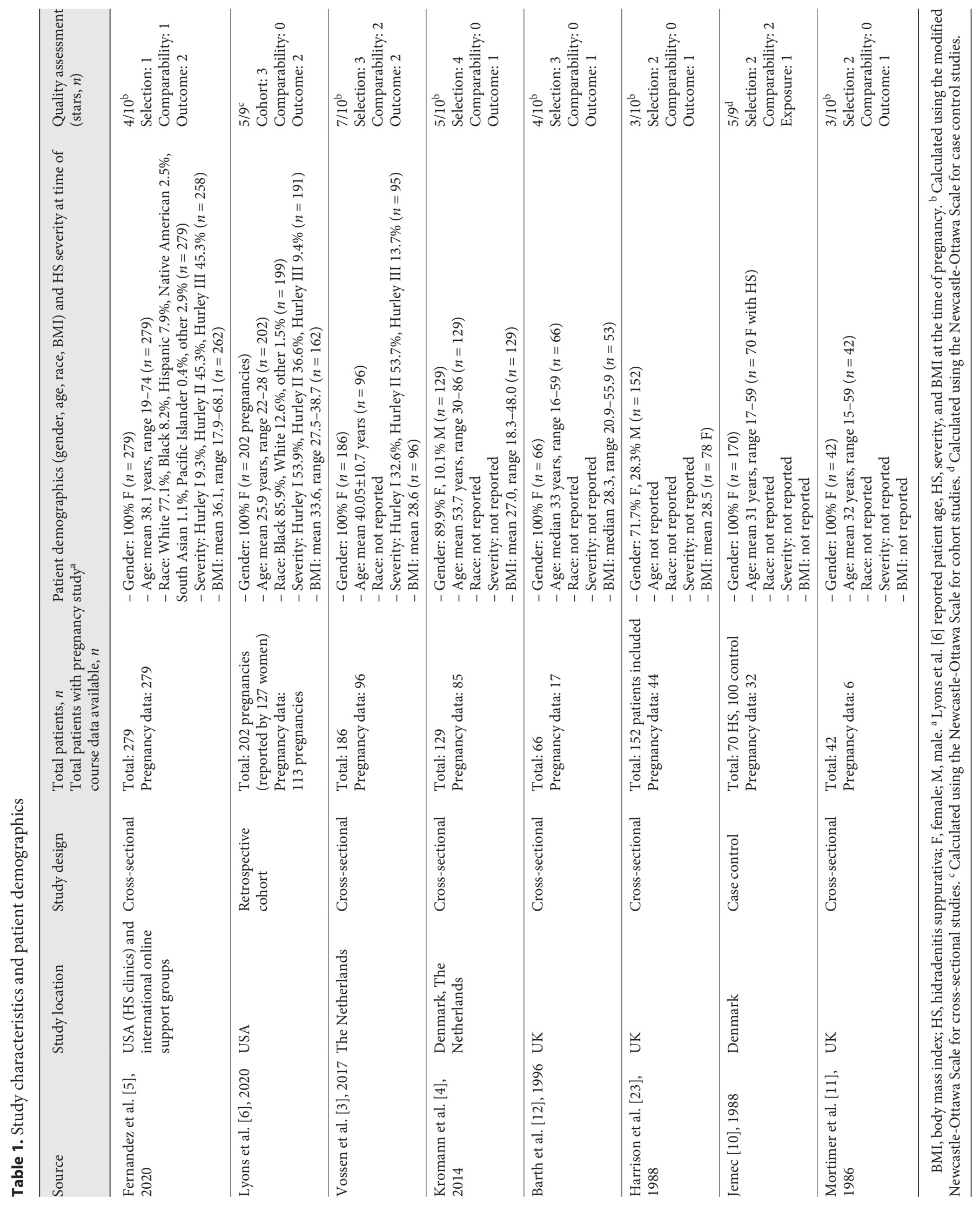




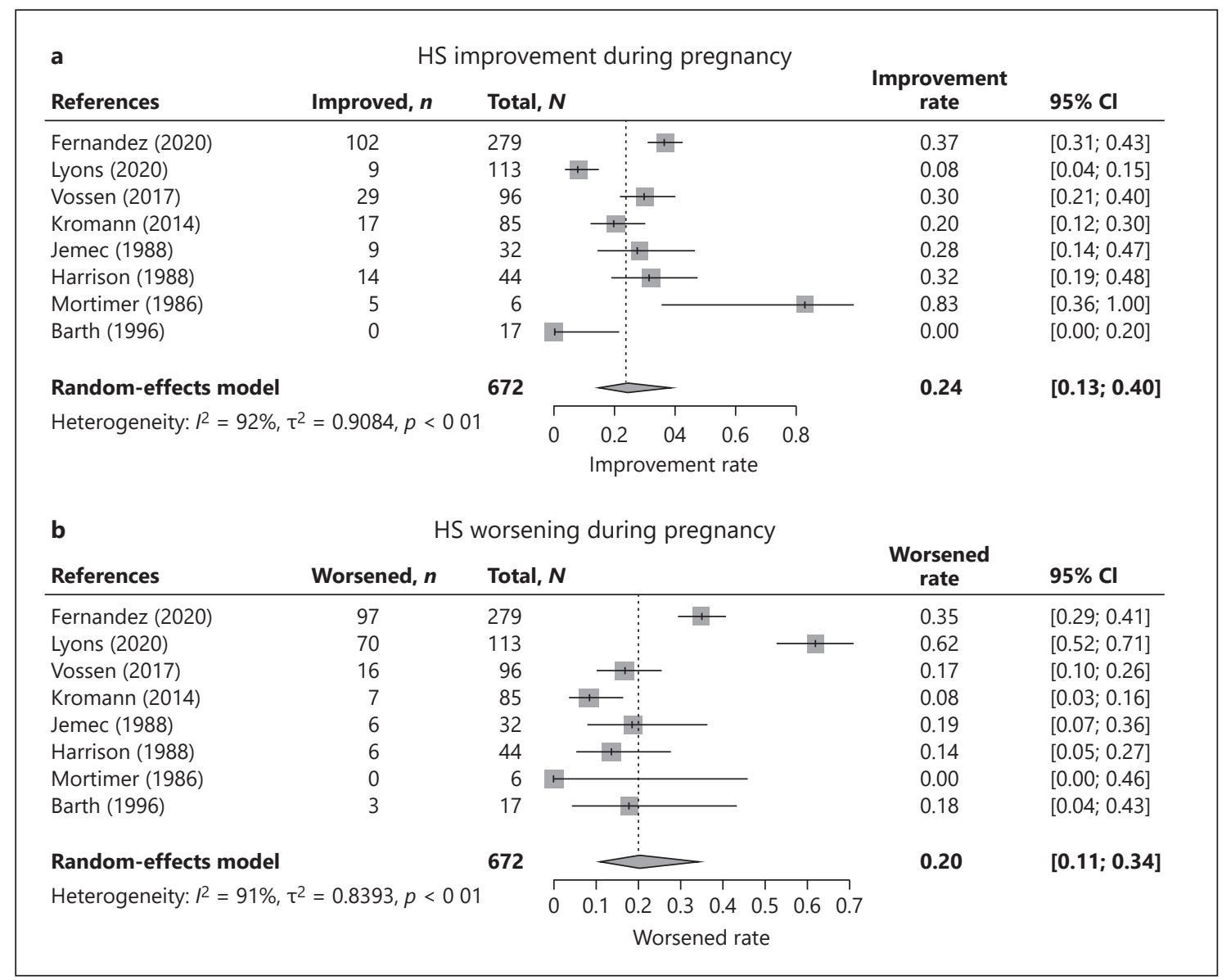

Fig. 2. a, b Forest plots of random-effects meta-analyses among pregnant HS patients. Eight studies on the HS disease course during pregnancy are shown. For each study, the square represents the observed rate with the whiskers estimating the $95 \%$ confidence interval. The dashed line represents the estimated pooled rate. The diamond at the bottom of each figure represents the overall pooled estimate of all studies along with the respective $95 \%$ confidence interval using the random-effects model.

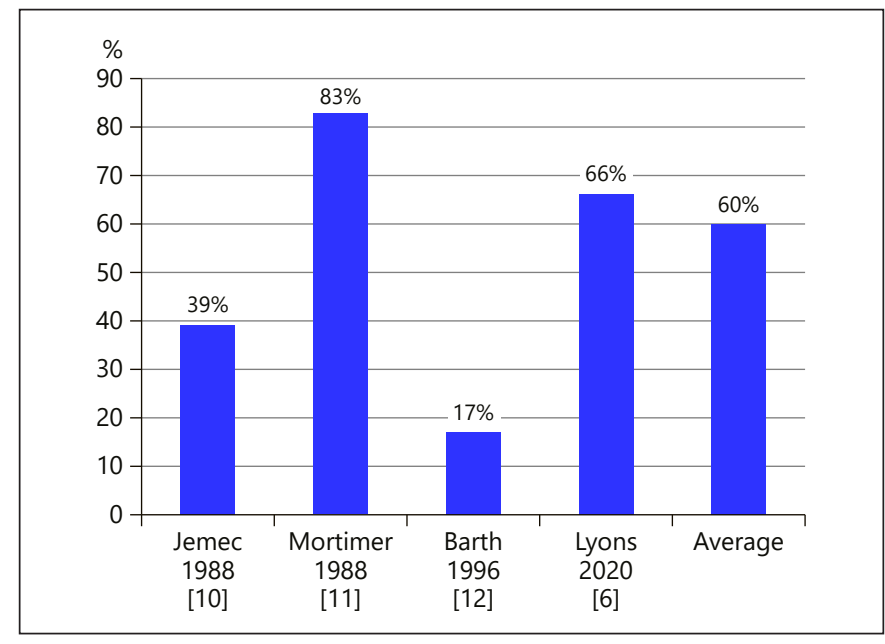

Fig. 3. Percentage of patients reporting postpartum HS flare.

\section{Discussion}

Our systematic review and meta-analysis found that the HS disease course during pregnancy and after delivery is variable; our random-effects meta-analysis showed a $24 \%$ rate of improvement of HS during pregnancy, and a 20\% rate of symptom worsening. Additionally, we found that $60 \%$ of women reported experiencing a postpartum disease flare. Previous literature characterizing the HS disease course during pregnancy has been limited by small sample sizes, retrospective designs, and lack of objective disease severity scoring measures. This meta-analysis studied a total of 672 pregnancy cases and 164 postpartum cases, providing a larger sample size and therefore a more robust estimate of HS disease activity changes during and after pregnancy than any of the included studies individually. 
Improvement in disease activity during pregnancy has been reported in autoimmune inflammatory diseases, including rheumatoid arthritis and multiple sclerosis [13, 14]. This improvement is thought to be secondary to an overall pregnancy-induced immunosuppressive state, driven by changes in $\mathrm{T}$ cell regulation, cytokine profiles, and hormonal influences on the immune system [15]. However, our study found that the effect of pregnancy on the HS disease course is mixed. This information is crucial to effectively counsel patients regarding their HS disease course during pregnancy, and to aid in planning potential treatment regimen modifications. Lyons et al. [6] found a high rate of HS exacerbation during pregnancy, but a lack of HS-directed medical treatment for these patients. Given our finding that most patients will remain stable or worsen, this emphasizes the importance of diligent monitoring by both dermatologists and obstetricians.

The impact of sex hormones on HS is evidenced by disease onset around puberty, worsening with menses, rare post-menopausal onset of disease [2], as well as an established association with polycystic ovarian syndrome [16]. Two studies included in our review examined the relationship between the presence of menstrual HS flares and HS disease activity during pregnancy. Fernandez et al. [5] found a positive correlation trending towards significance, wherein patients who had worsening during menses were more likely to have worsening during pregnancy $(p=0.06)$. Vossen et al. [3] identified a significant inverse relationship.

Pregnancy may impact HS in several different ways. Potentially protective factors for HS during pregnancy include immune modulation through changing levels of estrogen and progesterone. As estrogen levels rise during pregnancy, there is a shift from pro-inflammatory to antiinflammatory cytokines [17]; additionally, estrogen activates regulatory $\mathrm{T}$ cells, which have been correlated with improvement of other inflammatory diseases such as rheumatoid arthritis during pregnancy $[18,19]$. Progesterone exerts anti-inflammatory effects by suppressing interferon production and the activity of CD4+ T cells and $\mathrm{T}$ helper-17 cells, all of which are implicated in HS pathogenesis [20]. However, the specific effects of estrogen and progesterone on HS remains unclear, and some studies suggest that progesterone-laden treatments may worsen HS symptoms [21]. Further characterization of the timing of disease changes during pregnancy in relationship to hormone levels could provide additional insight into the mechanisms underlying hormonal impact on HS disease activity during pregnancy.

Hidradenitis Suppurativa Disease during Pregnancy
Potential contributing factors to HS flares during pregnancy include physical changes to the body, such as weight gain and increased friction in the intertriginous areas. Adipocytes have been shown to promote the secretion of pro-inflammatory cytokines including tumor necrosis factor- $\alpha$ [20]. Interestingly, Lyons et al. [6] found no correlation between weight gain during pregnancy and change in HS disease status. Furthermore, patients may need to discontinue certain medications prior to conception, such as oral contraceptives, spironolactone, or tetracyclines, which could precipitate disease flares during pregnancy.

The limitations of this study include that the data analyzed were largely obtained from survey studies and patient reporting of symptoms may involve recall bias. In addition, patients who volunteer to be part of HS survey studies may not be reflective of the general HS population. All of the included studies took place in North America and Europe, limiting generalizability. Only one study included information on HS treatment during pregnancy. Finally, substantial heterogeneity between studies was observed, likely due to differences in study design. Future investigations in this field should include improved reporting of pertinent demographics, medical complications of pregnancy, pregnancy history, objective measurements of disease activity, detailed timing of disease changes, defined criteria for postpartum HS flare, and inclusion of more diverse populations.

\section{Conclusion}

Although about a quarter of women with HS may experience improvement in their disease during pregnancy, the majority of women have a stable or worsened disease course. In addition, over half of women may have a postpartum disease flare. Close collaboration between dermatologists and obstetricians is needed to ensure appropriate monitoring for expecting HS patients. Large prospective pregnancy registries [22] may improve our understanding of the impact of pregnancy on HS. Finally, evidence-based consensus guidelines are urgently needed to aid in proper management for these patients.

\section{Key Message}

The majority of pregnant hidradenitis suppurativa patients experience stable or worsened disease and postpartum flare. 


\section{Acknowledgements}

Statistical analyses for this research was supported by NIH National Center for Advancing Translational Science (NCATS) UCLA CTSI grant No. UL1TR001881.

\section{Statement of Ethics}

IRB approval is not applicable to this study as all information was obtained from publicly available data and there was no handling of individual data.

\section{Conflict of Interest Statement}

J.L.H. has served as an advisor for Novartis. V.Y.S. is on the Board of Directors for the Hidradenitis Suppurativa Foundation, and has served as an advisor, investigator, and/or speaker for Sano- fi Genzyme, Regeneron, AbbVie, Burt's Bees, Dermira, Eli Lilly, Novartis, Pfizer, Galderma, Leo Pharma, SUN Pharma, Menlo Therapeutics, GpSkin, and Skin Actives Scientific. There was no financial transaction for the preparation of this manuscript. J.R.S., N.M.V., T.G., R.K.P., and A.M.T. report no conflicts of interest.

\section{Funding Sources}

No funding was received for this study.

\section{Author Contributions}

All authors contributed to drafting and revision of the manuscript and approved the final version for submission.

\section{References}

1 Garg A, Lavian J, Lin G, Strunk A, Alloo A. Incidence of hidradenitis suppurativa in the United States: a sex- and age-adjusted population analysis. J Am Acad Dermatol. 2017; 77(1):118-22

2 Riis PT, Ring HC, Themstrup L, Jemec GB. The role of androgens and estrogens in hidradenitis suppurativa - a systematic review. Acta Dermatovenerol Croat. 2016;24(4):23949.

3 Vossen AR, van Straalen KR, Prens EP, van der Zee HH. Menses and pregnancy affect symptoms in hidradenitis suppurativa: a cross-sectional study. J Am Acad Dermatol. 2017;76(1):155-6.

4 Kromann CB, Deckers IE, Esmann S, Boer J, Prens EP, Jemec GBE. Risk factors, clinical course and long-term prognosis in hidradenitis suppurativa: a cross-sectional study. $\mathrm{Br} J$ Dermatol. 2014;171(4):819-24.

5 Fernandez JM, Hendricks AJ, Thompson AM, Mata EM, Collier EK, Grogan TR, et al. Menses, pregnancy, delivery, and menopause in hidradenitis suppurativa: a patient survey. Int J Womens Dermatol. 2020;5(5): 368-71.

6 Lyons AB, Peacock A, McKenzie SA, Jacobsen G, Naik HB, Shi VY, et al. Evaluation of hidradenitis suppurativa disease course during pregnancy and postpartum. JAMA Dermatol. 2020;156(6):681-5.

7 Stang A. Critical evaluation of the NewcastleOttawa scale for the assessment of the quality of nonrandomized studies in meta-analyses. Eur J Epidemiol. 2010;25(9):603-5.
8 Herzog R, Álvarez-Pasquin MJ, Díaz C, Del Barrio JL, Estrada JM, Gil Á. Are healthcare workers' intentions to vaccinate related to their knowledge, beliefs and attitudes? A systematic review. BMC Public Health. 2013; 13(1):154.

9 Higgins JPT, Green S. Cochrane handbook for systematic reviews of interventions. London:Cochrane Collaboration; 2011.

10 Jemec GB. The symptomatology of hidradenitis suppurativa in women. Br J Dermatol. 1988;119(3):345-50.

11 Mortimer PS, Dawber RP, Gales MA, Moore RA. Mediation of hidradenitis suppurativa by androgens. Br Med J. 1986;292(6515):245-8.

12 Barth JH, Layton AM, Cunliffe WJ. Endocrine factors in pre- and postmenopausal women with hidradenitis suppurativa. Br J Dermatol. 1996;134(6):1057-9.

13 Jethwa H, Lam S, Smith C, Giles I. Does rheumatoid arthritis really improve during pregnancy? A systematic review and metaanalysis. J Rheumatol. 2018;46:245-50.

14 Finkelsztejn A, Brooks JB, Paschoal FM, Fragoso YD. What can we really tell women with multiple sclerosis regarding pregnancy? A systematic review and meta-analysis of the literature. BJOG. 2011;118(7):790-7.

15 Piccinni MP, Lombardelli L, Logiodice F, Kullolli O, Parronchi P, Romagnani S. How pregnancy can affect autoimmune diseases progression? Clin Mol Allergy. 2016;14:11.

16 Garg A, Neuren E, Strunk A. Hidradenitis suppurativa is associated with polycystic ovary syndrome: a population-based analysis in the United States. J Invest Dermatol. 2018; 138(6):1288-92.

17 Kassi E, Moutsatsou P. Estrogen receptor signaling and its relationship to cytokines in systemic lupus erythematosus. Poole B. J Biomed Biotechnol. 2010;2010:317452.

18 Nadkarni S, McArthur S. Oestrogen and immunomodulation: new mechanisms that impact on peripheral and central immunity. Curr Opin Pharmacol. 2013;13(4):576-81.

19 Hughes GC, Choubey D. Modulation of autoimmune rheumatic diseases by oestrogen and progesterone. Nat Rev Rheumatol. 2014; 10(12):740-51.

20 Perng P, Zampella JG, Okoye GA. Considering the impact of pregnancy on the natural history of hidradenitis suppurativa. Br J Dermatol. 2018;178(1):e13-4.

21 Collier EK, Price KN, Grogan TR, Naik HB, Shi VY, Hsiao JL. Characterizing perimenstrual flares of hidradenitis suppurativa. Int J Womens Dermatol.2020;6(5):372-6.

22 Adelekun AA, Micheletti RG, Hsiao JL. Creation of a registry to address knowledge gaps in hidradenitis suppurativa and pregnancy. JAMA Dermatol. 2020;156(3):353.

23 Harrison BJ, Read GF, Hughes LE. Endocrine basis for the clinical presentation of hidradenitis suppurativa. Br J Surg. 1988;75(10):9725.

24 Moher D, Liberati A, Tetzlaff J, Altman DG; PRISMA Group. Preferred reporting items for systematic reviews and meta-analyses: the PRISMA statement. PLoS Med. 2009 Jul 21; 6(7):e1000097. 